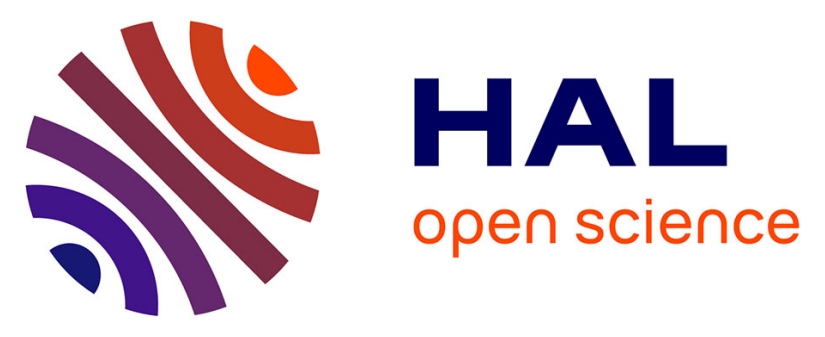

\title{
Hybrid Plasmonic Mode by Resonant Coupling of Localized Plasmons to Propagating Plasmons in a Kretschmann Configuration
}

Mitradeep Sarkar, Mondher Besbes, Julien Moreau, Jean-François Bryche, Aurore Olivéro, Grégory Barbillon, Anne-Lise Coutrot, Bernard Bartenlian, Michael Canva

\section{To cite this version:}

Mitradeep Sarkar, Mondher Besbes, Julien Moreau, Jean-François Bryche, Aurore Olivéro, et al.. Hybrid Plasmonic Mode by Resonant Coupling of Localized Plasmons to Propagating Plasmons in a Kretschmann Configuration. ACS photonics, 2015, 2 (2), pp.237-245. 10.1021/ph500351b . hal01307382

\section{HAL Id: hal-01307382 \\ https://hal-iogs.archives-ouvertes.fr/hal-01307382}

Submitted on 6 Oct 2017

HAL is a multi-disciplinary open access archive for the deposit and dissemination of scientific research documents, whether they are published or not. The documents may come from teaching and research institutions in France or abroad, or from public or private research centers.
L'archive ouverte pluridisciplinaire HAL, est destinée au dépôt et à la diffusion de documents scientifiques de niveau recherche, publiés ou non, émanant des établissements d'enseignement et de recherche français ou étrangers, des laboratoires publics ou privés. 


\title{
Hybrid plasmonic mode by resonant coupling of localized plasmons to propagating plasmons in a Kretschmann configuration
}

\author{
Mitradeep Sarkar ${ }^{1}$, Mondher Besbes ${ }^{1}$, Julien Moreau ${ }^{1}$, Jean-François Bryche ${ }^{1,2}$, \\ Aurore Olivéro ${ }^{1}$, Grégory Barbillon ${ }^{2}$, Anne-Lise Coutrot ${ }^{1}$, Bernard Bartenlian ${ }^{2}$, \\ Michael Canva ${ }^{1 *}$ \\ 1 - Laboratoire Charles Fabry, \\ Institut d'Optique Graduate School, Univ Paris Sud, CNRS, \\ 2 avenue Augustin Fresnel, 91127 Palaiseau, France \\ 2 - Institut d'Électronique Fondamentale, \\ Université Paris Sud, CNRS \\ Bât. 220, - Rue Ampère, 91405 Orsay, France
}

\begin{abstract}
Metal nanoparticles have the ability to strongly enhance the local electromagnetic field in their vicinity. Such enhancement is crucial for biomolecular detection and is used by techniques such as surface plasmon resonance detection or surface enhanced Raman scattering. For these processes, the sensitivity strongly depends on the electromagnetic field intensity confined around such nanoparticles. In this article, we have numerically studied an array of metallic nanocylinders, which can sustain Localized Surface Plasmons (LSP). However, the excitation wavelengths of the LSP are not tunable due to their limited dispersion. We have demonstrated a plasmonic mode, the Hybrid Lattice Plasmon (HLP), which is excited in such a periodic array by adding a uniform thin metallic film below it. This mode is a result of a harmonic coupling between the propagating surface plasmons present in such a metallic film with the Bragg waves of the array. It shows a strong confinement of the electromagnetic field intensity around the nanocylinders, similar to the LSP, but the dispersion of this HLP mode is, however, similar to that of the propagating plasmons, and thus can be tuned over a wide range of excitation wavelengths. The structure was fabricated using electron beam lithography, and characterized by a surface plasmon resonance setup. These experimental results show that the HLP mode can be excited in a classical Kretschmann configuration with a dispersion similar to the prediction of numerical simulations.
\end{abstract}

KEYWORDS. Plasmonics, Nano-materials, Field enhancement, surface plasmon resonance modes, hybrid plasmon 
Metal nanoparticles have been extensively studied due to their ability to enhance local electromagnetic field in their vicinity. ${ }^{12}$ Such enhancement results from the confinement of the field at nanoscale by surface plasmon resonances (SPR) in metallic structures. When light is incident on a metallic structure, the photons can resonantly couple to the collective oscillations of free electrons at the structure surface resulting in a surface plasmon polariton.

In a uniform metallic film, propagating surface plasmon (PSP) can be excited at the interface of the metal and the dielectric resulting in an electromagnetic (EM) wave propagating along the interface. These PSP are evanescent waves with a typical penetration depth in the dielectric of about $200 \mathrm{~nm}$ and are widely used for optical label free SPR biosensing. ${ }^{3}{ }^{5}$ To excite PSP, it is necessary to match its momentum to the incident photon momentum. SPR sensors use either prism coupling (Kretschmann or Otto configurations), ${ }^{67}$ grating coupling ${ }^{89}$ or waveguide coupling ${ }^{10}$ to couple the incident radiation to SPPs.

On the other hand, metallic nanostructures with dimensions less than the wavelength of the incident light can sustain localized surface plasmon (LSP) that do not propagate. The local charge oscillations at the structure edges lead to an intense local field at the metal dielectric


nanostructures has been used for a number of applications, such as Surface Enhanced Raman Scattering (SERS), ${ }^{14}{ }^{15}$ drug delivery, chemical sensing, cancer therapy, and new photonic devices. ${ }^{161718}$

For all the above-mentioned applications, it is important to have a high EM field confinement close to the metal surface. ${ }^{19} 2021$ This can be achieved with nanostructures of dimensions smaller than the wavelength of light, to effectively confine the electromagnetic field and thus enhance the sensor performance for detection of trace concentrations of bio-molecules. In particular, there 
has been a surge of interest in studying the effect of coupling between plasmon polaritons. It is well known for example that coupling of plasmons between nanostructures can sometimes result in some high field confinements in the gap between them. ${ }^{22} 23242526$ Indeed, when the structure is illuminated from the nanostructure side, the Bragg waves of the array give rise to a hybrid plasmonic mode. ${ }^{27} 28{ }^{29}$ This Bragg mode (BM) can help to confine the EM field around the nanostructures. Also, periodic array of nanostructures, when excited in the Kretschmann configuration, can support surface lattice resonances (SLRs), which are caused by the coupling of the diffracted orders of the array to the LSP. ${ }^{30} 3132$ However, for periods much smaller than the incident wavelength, such BMs and SLRs do not have much of an effect. For an efficient excitation of these modes, structures with larger dimensions are needed but the confinement of the EM field in such large structures is generally weak. Also, such resonances are restricted within a very small range of excitation wavelengths and cannot be tuned for various applications.

In this paper, we show that the introduction of a metallic film, below a nanostructure array, which can sustain PSP, can significantly enhance the field around the nanostructures for small array dimensions. In certain configurations, the PSPs can be harmonically coupled to the Bragg wave of the array. This modifies the properties of the PSP, giving rise to a hybrid lattice plasmon (HLP) mode with a large dispersion and an enhancement of the local EM field around the metallic nanostructures. This type of hybrid mode occurs whenever there is a coupling between two different plasmons 333435 and they have appeared in the literature from time to time as Hybrid Plasmons 36373839 or Fano-like resonances. ${ }^{4041} 4243$ However, the HLP mode is different from the Fano-like resonances as it results from the coupling of two plasmonic modes, which can be expressed as "broad states", and not from the coupling of a broad and discrete state for Fano 
resonances. This coupling is largely dependent on the periodicity of the array and is in some sense similar to the modes found in photonic crystals. ${ }^{44} 45$

Though similar modes and mechanisms of excitation have been studied before, but their excitations in the Kretschmann configuration and the field enhancement, which results from such coupling, have not been explored with respect to biosensing. We will show that the magnitude of the field intensity of this HLP mode is much greater than with the BM and the SLR. Also, almost all previous studies used a dielectric spacer layer between nanostructures and the metallic film, which would not be ideal for biosensing as most of the field would then be concentrated in the spacer layer, and not around the particles, where the biomolecules would actually be adsorbed.

In this paper, we study numerically such coupling of PSP with other excited modes in a periodic array of metallic nanocylinders. A simple mechanism is given to explain the features observed in the absorption map. The electrical field intensity distribution of the different modes is also discussed. Finally, these numerical predictions are compared to experimental results showing the existence of the HLP mode.

\section{RESULTS AND DISCUSSIONS}

We have used gold cylinders as metallic nanostructures with a period of the array $(\Lambda)$ of 200 $\mathrm{nm}$ and a cylinder diameter (D) of $50 \mathrm{~nm}$. The cylinder heights as well as the heights of the uniform gold layer below the cylinder array were varied to study their effect. We have chosen the conventional Kretschmann configuration with a BK7 glass prism to couple the incident light to the PSP. However, we must mention that the new modes and the explanations of their origin presented in this article also hold for other methods used to couple the incident light to the PSP. 
The medium surrounding the cylinders is water, keeping in mind the applications of these structures to biomolecular detections. The structure is shown in Figure 1.

To get a better understanding on what happens when the incident light couples to this structure, we will start by briefly showing the various plasmonic modes that exist in an uniform metallic film and then in a nanostructure array on glass substrate when they are excited in the Krestchmann configuration. We will also briefly mention, for the sake of comparison, the modes in the structure when excited from the nano-structure side.



Figure 1. Structure geometry consisting of a gold cylinder array with a diameter of $50 \mathrm{~nm}$, a period of $200 \mathrm{~nm}$, and a height $h_{2}$. A gold film of height $h_{1}$ is placed below the array. The medium above the gold film is water and the medium below is glass (BK7). TM polarized field is incident on the structure at an angle $\theta$ and azimuthal angle $\phi=\pi / 2$. The HLP mode is excited in the structure by coupling the PSP and the BM mode.

\section{Plasmonic modes in a uniform metallic film and a nanocylinder array on glass substrate.}

For a uniform metallic layer the wave-vector of the propagating surface plasmon (PSP) in the plane parallel to the metal-dielectric interface $\left(k_{x}\right)$ is given by the well-known equation ${ }^{46}$

$$
k_{p s p}=k_{0} \sqrt{\frac{n_{d}^{2} n_{m}^{2}}{n_{d}^{2}+n_{m}^{2}}}
$$


where $k_{0}=2 \pi / \lambda$ is the free space wave vector for the wavelength $\lambda, n_{m}$ and $n_{d}$ are the refractive indices of the metal and the medium above the metal surface, respectively. This mode exists only for the TM polarized light because a non-zero component of the incident electric field perpendicular to the metal-dielectric interface is necessary for the excitation. The resulting dispersion for a uniform gold film is shown in Figure 2(a) for a height $h_{1}$ of $60 \mathrm{~nm}$. The PSP exist for values of the in-plane wave-vectors $\left(k_{x}=k_{0} n \sin \theta\right.$ where $n$ the refractive index of the medium) that lie above the critical angle for the BK7-water interface (lightline in the medium above the metal surface with $\left.k_{x} / k_{0}=n_{d}\right)$, and below the lightline in BK7 $\left(k_{x} / k_{0}=n_{b}\right.$ (refractive index of glass)). We have chosen the representation in the $\left(n_{\text {eff }}, k_{0}\right)$ space where $n_{\text {eff }}=k_{x} / k_{0}=$ $n_{b} \sin \theta$.

On the other hand, the absorption of nanostructures can be calculated using the Mie theory and dipolar polarizability to give the resonance condition for the LSPs, but such theories apply only for spherical or elliptical structures ${ }^{47} 1148$. However we have used exact numerical calculation to characterize a single and an array of nanocylinders in the Kretschmann configuration. The absorption for two different heights of nanocylinders $\left(\mathrm{h}_{2}\right)$ is shown in Figures $2(\mathrm{~b})$ and 2(c). The localized surface plasmon resonance modes can be excited for both the TM and TE polarized incident light. The resonance of the LSPs excited in such structures has almost no dispersion, as these are non-propagating plasmons. It can be seen that the absorption of the array matches closely that of single structure.

The slight red shift of the frequency with narrowing of the resonance linewidth for higher $k_{x}$ (above the critical angle of glass water interface) can be attributed to the SLR phenomenon as mentioned elsewhere. ${ }^{49} 50$ The SLR mode is excited when the Bragg vector (given by $k_{B}=$ $2 \pi / \Lambda$ for a structure with period $\Lambda$ ) matches the in-plane wave-vector $\left(k_{x}\right)$ caused by the 
diffraction orders from the nanostructure array. The condition for such constructive interference can be written as $k_{x} \pm \mathrm{m} k_{B}=k_{0} n$ where $\mathrm{m}$ is an integer, and $n=n_{b}$ if the condition is fulfilled in glass (in reflection) or $n=n_{d}$ when considered in water (in transmission). We see that the dispersion of the cylinders follows the SLR condition.

However, the dispersion of the SLR is insignificant when compared to the dispersion of the PSP or the subsequent modes that we studied. The range of wavelengths where the SLR mode can be effectively excited does not change significantly by changing the cylinder dimensions (height $\mathrm{h}_{2}$ ) as can be seen in Figures 2(b) and 2(c). The SLR mode is excited by the in-plane wave-vectors due to diffraction, which depend on the structure periodicity and not on cylinder height. 



Figure 2. (a) Normalized absorption (A) as a function of $k_{0}$ and $n_{\text {eff }}=k_{x} / k_{0}$ in a uniform gold film placed on a BK7 substrate with a height of $h_{1}=60 \mathrm{~nm}$. The PSP dispersion as calculated by Eq. 1 is also shown (black dashed-dotted). (b-c) Absorption maps for an array of cylinders of $50 \mathrm{~nm}$ in diameter with a period of $200 \mathrm{~nm}$ placed on a BK7 substrate for two values of the cylinder height (b) $h_{2}=30 \mathrm{~nm}$, and (c) $h_{2}=60 \mathrm{~nm}$. For comparison, the absorption curve for a single cylinder as a function of $k_{0}$ is also shown (blue line) as well as the fulfillment of the SLR condition in BK7 (black dashed) and in water (black solid).

Excitation of the HLP mode in the array of nanocylinders on a metallic film. Finally, the absorption (A) for the different modes present in the complete structure (Figure 1) is shown in Figure 3, for an incident TM polarized light, and two different heights of the nanocylinder in the Kretschmann configuration. For TE polarized light, no modes exist in the system (data not shown). 

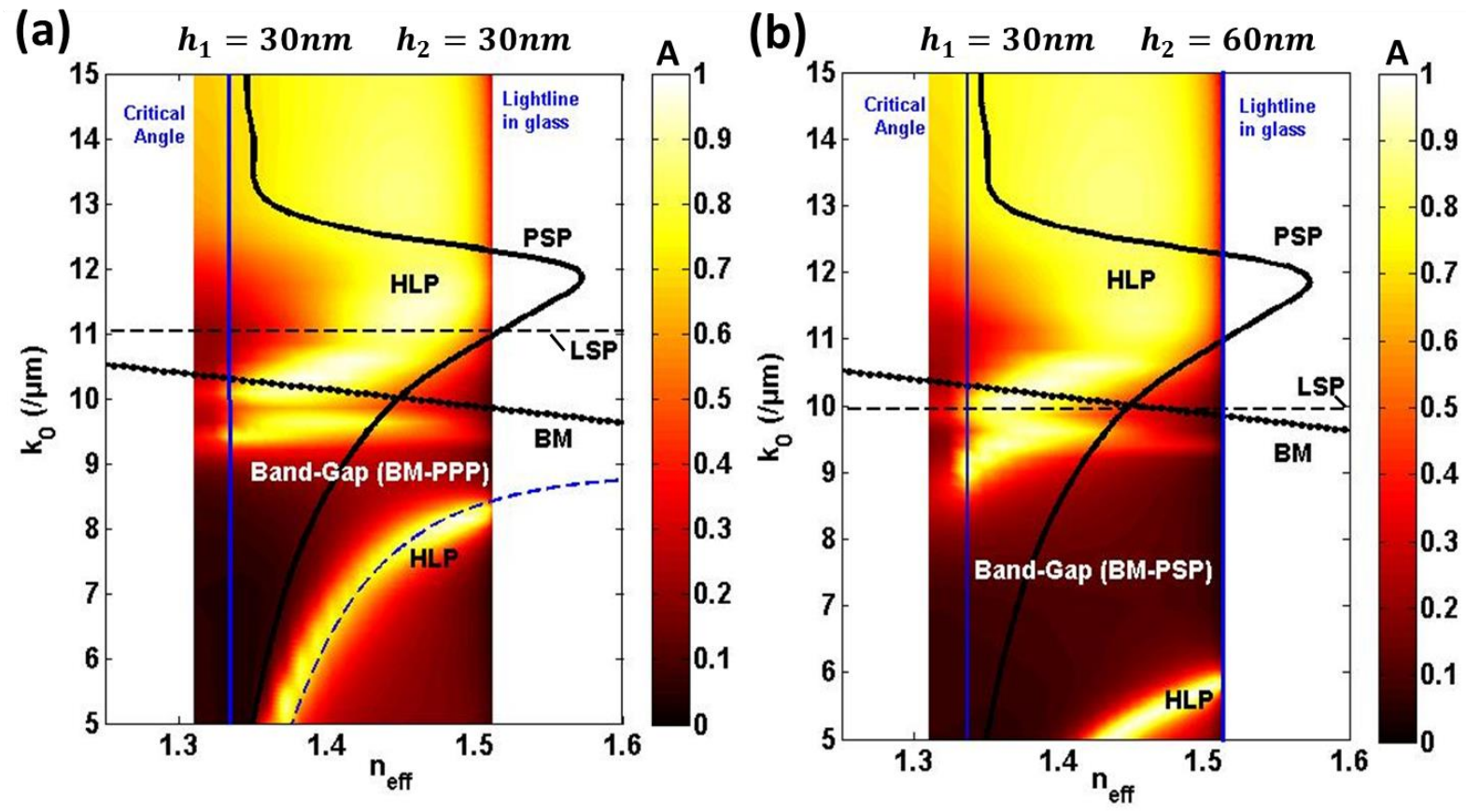

Figure 3. Normalized absorption (A) of the structure as a function of $k_{0}$ and $n_{\text {eff }}=k_{\mathrm{x}} / k_{0}$ for $\mathrm{h}_{1}$ $=30 \mathrm{~nm}$ and $\mathrm{h}_{2}=30 \mathrm{~nm}$ (a), and $60 \mathrm{~nm}$ (b). The dispersion curves for the PSP and BM as calculated by Eq.1 and Eq.2, respectively, are shown. The resonance frequency of the LSP was calculated using the FEM method for a single cylinder. The dispersion for the HLP mode calculated using Eq. 3 is also shown for $\mathrm{h}_{2}=30 \mathrm{~nm}$ (blue dashed).

The presence of the mode marked as BM (Bragg Mode) can be explained as follows: the PSP in the thin film is partially reflected by the edges of the nanostructure array. Thus, we have two contra-propagating PSP modes with the same in plane wave vector. The Bragg condition ${ }^{52}$ for the constructive interference of such contra-propagating modes can be written as

$$
k_{B M}=k_{p s p} \pm m k_{B}
$$

where $m$ is an integer. $k_{B}$ is the Bragg wave vector given by $2 \pi / \Lambda$ for the structure with period $\Lambda$. When the Bragg condition is fulfilled, a localized plasmonic mode is obtained with a field confined around the cylinders. We see in Figure 3 that the dispersion of the BM resonance follows closely the fulfillment of the Bragg condition calculated in Equation 2 with $\mathrm{m}=1$. A little shift from Equation 2 may be expected as the true values of $k_{p s p}$ are not exactly as that 
predicted in Equation 1 for lower heights of the gold film. The shift can also be the result of the coupling between the BM and the individual localized plasmonic mode. The excitation of this mode follows the same principle as that of the SLR mode mentioned earlier. The difference lies in the fact that in the case of the BM, the energy comes from the propagation plasmons (PSP), while for the SLR it originates from the diffraction orders from the structure. We will henceforth use the nomenclature 'SLR' for the mode excited in the nanostructure array on glass substrate and 'BM' for that with the nanostructure array on the gold film.

Another striking feature of the absorption map is the two modes marked in Figure 3 as HLP, which have a similar dispersion as the PSP modes shown in Figure 2(a). These modes are excited by the PSP present in the thin gold film and this is further verified by the fact that these modes do not exist for TE polarized incidence. To get a better understanding of this HLP mode, we can consider the structure as a combination of two harmonic oscillators namely the PSP, and the BM. It is well known ${ }^{53}$ that such a coupling of harmonic oscillators results in the characteristic anticrossing phenomenon and a frequency splitting. ${ }^{54} 55$ In Figure 3, we observe such a frequency splitting with the two modes on both sides of the resonance frequencies of the BM mode. The characteristic frequencies of two coupled harmonic oscillators can be written as:

$$
\omega_{H L P}^{\mp}=\frac{1}{2}\left(\omega_{p s p}^{2}+\omega_{B M}^{2}+2 K \mp \sqrt{\left(\omega_{p s p}^{2}-\omega_{B M}^{2}\right)^{2}+4 K^{2}}\right)
$$

where $\mathrm{K}$ is the coupling coefficient, $\omega_{p s p}$, and $\omega_{B M}$ are the resonance frequencies of the PSP and BM, respectively. The dispersion of the lower branch of the HLP mode using Equation 3 is shown in Figure 3. We see it closely follows the results obtained from the rigorous calculations which validate our interpretation of the origin of the HLP mode.

Now, when the BM mode resonance lies close to the resonance frequencies of the LSP mode as for the case of cylinder height of $60 \mathrm{~nm}$ in Figure 3, we have a coupling between 3 modes 
namely the PSP, BM, and LSPR. In such a situation, the photonic bandgap can be expected to increase and the frequencies of the HLP mode moves further away from the frequencies of both the PSP, and BM. We see such a broadening of the bandgap in Figure 3 for $h_{2}=60 \mathrm{~nm}$. Such a coupling could be analytically expressed using a $3 \times 3$ coupling matrix, but with some unknown coupling parameters.

Effect of different heights on the HLP mode position. The effects of $h_{1}$ (gold film height) and $\mathrm{h}_{2}$ (cylinder height) were studied. Firstly, the resonance frequencies of the modes are not expected to change with $\mathrm{h}_{1}$ as it only affects the PSP mode and its dispersion is largely independent of the film height. Indeed, this is observed on Figure 4(a). On the contrary, the position of the HLP mode strongly varies with the cylinder height (Figure 4(b)). This is explained by the coupling between the BM and PSP modes, which is stronger when the cylinder height is increased, thus pushing the HLP mode to higher wavelengths (lower $k_{0}$ ). Also the LSP mode shifts to higher wavelengths with increasing height as can be seen in Figure 2(b-c) and this further causes a red-shift of the HLP. We see a somewhat linear dependence of the resonance wavelength of the HLP mode and the cylinder height (Figure 4(c)). This gives the opportunity to tune the resonance wavelength of the HLP mode, over a range of $1000 \mathrm{~nm}$, depending on the application, by changing the cylinder height $\left(\mathrm{h}_{2}\right)$ while retaining the same high field intensity distribution as seen later. 


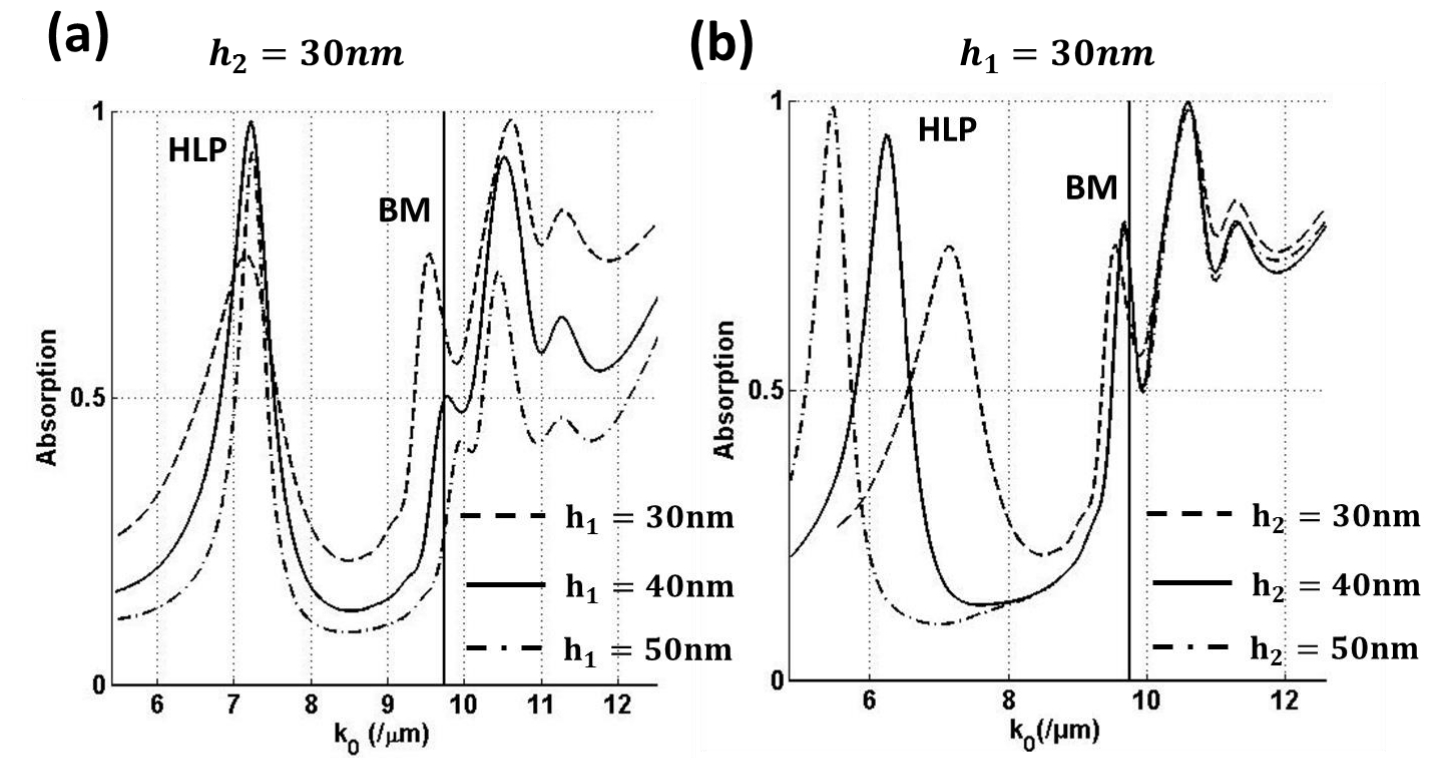

(b) $\quad h_{1}=30 \mathrm{~nm}$

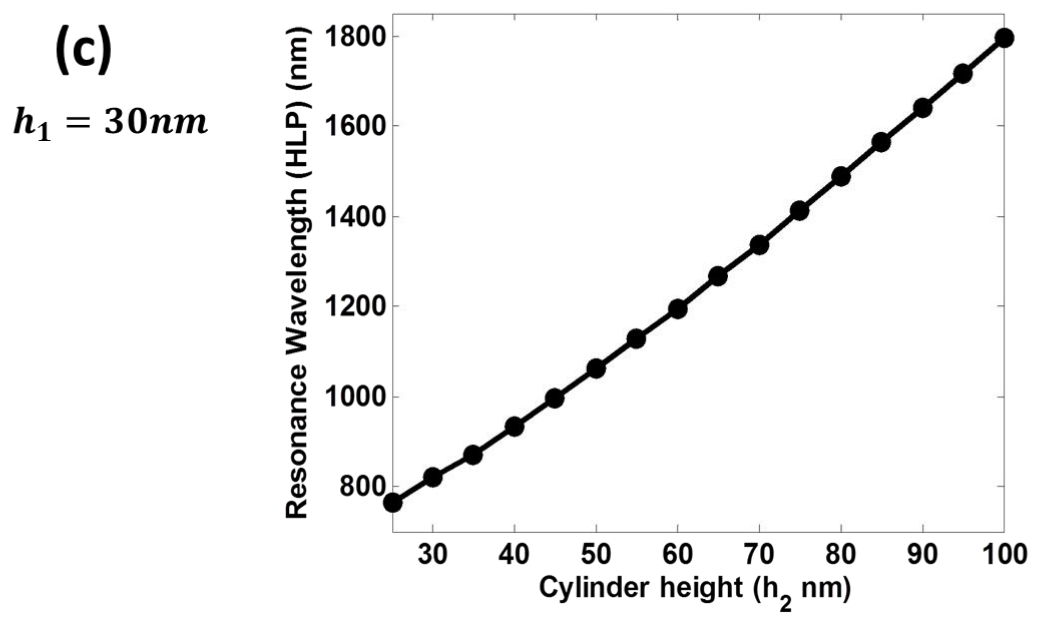

Figure 4. (a) Normalized absorption curve of the HLP mode as a function of $k_{0}$ for three different heights of the gold film $\left(h_{1}\right)$ with fixed cylinder height $\left(h_{2}=30 \mathrm{~nm}\right)$. The curves are shown at $n_{\text {eff }}=1.42$ (b) same for three different cylinder heights with a gold film height fixed at $\mathrm{h}_{1}=30 \mathrm{~nm}$. The vertical solid lines represents the calculated BM position using Eq.2 at $\mathrm{n}_{\mathrm{eff}}=1.42$.(c) Resonance Wavelength of HLP mode for $\mathrm{h}_{1}=30 \mathrm{~nm}$ with different cylinder heights $\left(\mathrm{h}_{2}\right)$.

Electric field intensity distribution of modes. The electric field intensity distributions $(\mathrm{I}=$ $\mathrm{E}_{\mathrm{x}}{ }^{2}+\mathrm{E}_{\mathrm{y}}{ }^{2}+\mathrm{E}_{\mathrm{z}}{ }^{2}$ ) normalized to the incident field intensity $\left(\mathrm{I}_{0}\right)$, at each point over one period of the structure for the 3 modes (HLP and BM) are shown in Figure 5. For all modes, the field component orthogonal to the metal surface is the strongest. 




Figure 5. Normalized intensity distribution $\left(\mathrm{I} / \mathrm{I}_{0}\right)$, in logarithmic scale, of the different modes over one half-period $(200 \mathrm{~nm})$ of the array for $\mathrm{h}_{1}=30 \mathrm{~nm}$ and $\mathrm{h}_{2}=30 \mathrm{~nm}$. The nanocylinder is located at $x=0, y=0$, and $z=0$. The intensity was calculated for different values of $k_{0}$,

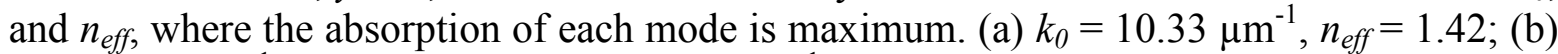
$k_{0}=9.60 \mu \mathrm{m}^{-1}, n_{\text {eff }}=1.46$; (c) $k_{0}=7.20 \mu \mathrm{m}^{-1}, n_{\text {eff }}=1.42$. The positions on the $\left(k_{0}, n_{\text {eff }}\right)$ absorption map, where the fields were calculated, are shown as dots.

For the lower frequency branch of the HLP mode, we see a strong confinement of the field around the cylinder top with a symmetric distribution. This is similar to the field distributions we expect in dipoles or for LSP mode. We therefore have a field distribution similar to LSP modes and a dispersion similar to PSP modes, which further validates the fact that this mode is a result of coupling between the two. For BM mode, we have an asymmetric intensity distribution with more field intensity concentrated on the cylinder edge facing the direction of incidence. A closer look at the metal film surface $(z=0)$ shows a weak sinusoidal variation of the intensity along the 
axes of the PSP propagation ( $x$-axis), which is expected from the standing wave formed by the interference of the contra propagating PSPs. Finally, for the higher frequency branch of the HLP mode, we have a somewhat orthogonal distribution to that of its lower frequency branch with the field intensity being concentrated around the bottom edge of the cylinders.

We then compare the intensity distribution of the HLP mode (lower frequency branch) to that of the classical resonance mode (SLR) in a cylinder array without gold film. Figure 6 shows, in the plane of incidence ( $x-z$ plane), the intensity variation at a distance of $1 \mathrm{~nm}$ from the surface with respect to curvilinear coordinates. The fact that the field intensity distribution is not exactly symmetric with respect to the nanostructure geometry is due the direction of the propagating plasmons. Both the SLR and the HLP modes have much higher field intensity than the BM, which is also the mode we expect, when the structure is excited from the nanostructure side (see supplementary data). This validates the benefit of illuminating the structures in the Kretschmann configuration as compared to excitation from the nanostructure side. It is clear that the HLP mode obtained with a gold film below the cylinder array gives the highest field intensity on the nanostructure top surface. Both SLR and HLP modes show some very high intensity peaks predicted by the numerical model at the top and bottom corners of the structure which will probably not be observed as such, first because their exact amplitude depends on the mesh finesse used in the numerical simulation and also because in a real sample, the edges will not be perfect. However, we have carefully checked that the value of the integral of intensity as calculated in the next section, which is the important quantity for biomolecular detection, is largely independent of the mesh finesse. 


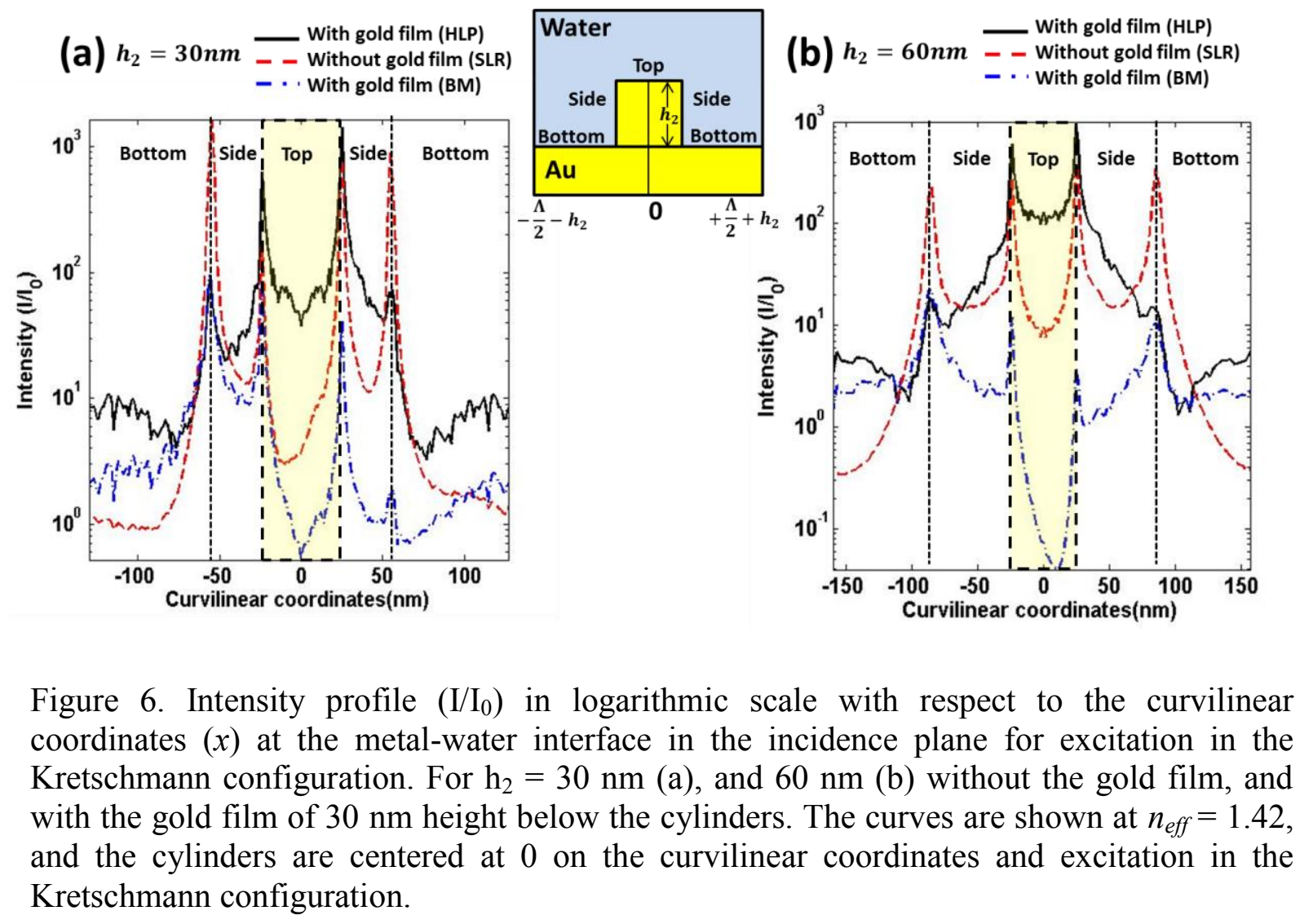

Mean intensity enhancement. As we are mainly interested in the overall sensitivity gain due to the nanostructure, we have calculated the surface integral of the intensity distribution at a distance of $1 \mathrm{~nm}$ from the surface of the cylinders $\left(S_{c y l}\right)$ as

$$
I_{S}=\frac{1}{S_{c y l}} \iint_{S_{c y l}} I(S) d S
$$

The dependence of the integrated intensity on the effective refractive index $\left(n_{\text {eff }}\right)$ for the HLP and SLR mode is shown in Figure 7. As before, it was calculated for a value of $h_{1}=30 \mathrm{~nm}$ and shown for two values of $h_{2}(30 \mathrm{~nm}$ and $60 \mathrm{~nm})$. We can see that the mean intensity around the cylinders is enhanced up to 3 times when using the HLP mode compared to the classical SLR mode in a nanostructure array. Such an enhancement can prove to be very useful in SPR applications, where the sensitivity strongly depends on the field intensity ${ }^{56}$, and even more for 
SERS ${ }^{57}$ where the signal is proportional to the surface integral of $\mathrm{I}^{2}$. It can be shown that we have an enhancement up to 9 times for $\left[\iint \mathrm{I}^{2}(\mathrm{~S}) \mathrm{dS}\right] / \mathrm{S}_{\mathrm{cyl}}$ obtained with the HLP mode as compared to the SLR mode (see Supplementary data). Thus such a structure can be effectively used as a substrate for both the processes. ${ }^{58} 7$ The HLP mode also has a mean intensity higher by an order of magnitude (and surface integral of $\mathrm{I}^{2}$ up to $10^{2}$ times) as compared to that of the BM (see Supplementary data). Applications that require high field intensity can therefore take advantage of the HLP mode in the Kretschmann configuration as compared to using the BM.



Figure 7. (a) Integrated intensity $I_{S}$ with $h_{2}=30 \mathrm{~nm}$, with and without a $30 \mathrm{~nm}$ gold film, as a function of $n_{\text {eff. }}$ (b) Same for $\mathrm{h}_{2}=60 \mathrm{~nm}$. The excitation is in the Kretschmann configuration.

For biosensing applications, the biomolecules may be difficult to localize selectively only around the nanocylinders, and an integration of the field intensity over the entire unit period of 
the array may be more useful to estimate the performance of the structures. We have observed that the field enhancement of the HLP mode with respect to the SLR mode over the entire surface of one structure period is a bit higher than that shown in Figure 7 (see Supplementary data). Because the HLP mode results from the coupling of propagation plasmons and has a higher field distribution far from the cylinders than the SLR mode (Figure 6), we can expect a higher field intensity enhancement for the HLP mode than the SLR mode when the entire surface of an unit period of the structure is taken into account.

Experimental results. The nanostructure shown in Figure 1, with $h_{1}=30 \mathrm{~nm}$ and $h_{2}=30 \mathrm{~nm}$, was fabricated by Electron Beam Lithography in order to have a good control of the size, shape and distance between nanocylinders. A thin layer of $3 \mathrm{~nm}$ titanium was used for adhesion of the gold film on the BK7 substrate. SEM images (Figure 8(a)) shows that the resulting gold nanocylinders have a mean diameter of around $50 \mathrm{~nm}$ and a periodicity of $200 \mathrm{~nm}$. The structure was characterized by a SPR imaging system based on spectral scanning modality similar to a previously reported setup (see Supplementary data). ${ }^{69}$ Here, the normalized reflectivity (R) (TM reflectivity divided by TE reflectivity) of the sample is measured as a function of the incident wave vector $k_{0}$ and effective index $n_{\text {eff. }}$ For the numerical simulation, the Titanium adhesion layer below the gold film was also taken into consideration. As can be seen in Figure 8(b), a good agreement between the experimental results and the exact numerical simulation is obtained for the HLP mode and thus this mode can be effectively excited using the Kretschmann setup. The BM is somehow weaker in the experiment than what is predicted by the simulation. As can be seen from Figure 4(a), the absorption of the BM strongly depends on the gold film height which may be lower than expected in the fabricated structure. 


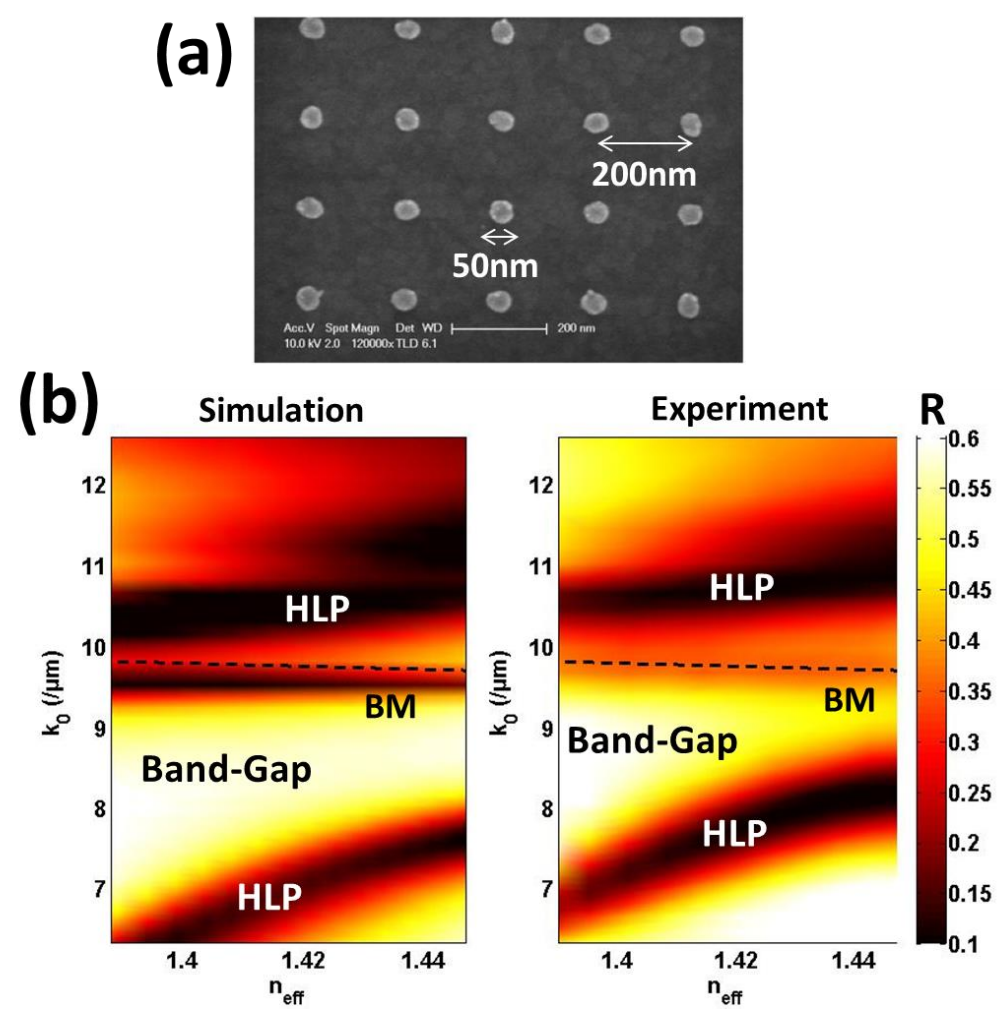

Figure 8. (a) SEM image of gold cylinders with a diameter of $50 \mathrm{~nm}$, a period of $200 \mathrm{~nm}$, and a height of $30 \mathrm{~nm}$ on a $30 \mathrm{~nm}$ gold film. (b) Reflectivity (R) of the structure as a function of $k_{0}$, and $n_{\text {eff }}=k_{x} / k_{0}$ calculated by numerical simulation, and measured experimentally. The position of the BM mode, as calculated by Equation 2, is also shown (dotted).

\section{CONCLUSION}

In summary, we have numerically calculated the absorption as a function of excitation wavelength, and incident internal angle for an array of metallic nanocylinders with and without an underlying thin metallic film in the Kretschmann configuration. We have shown that mean field intensity around the array of nanostructures can be enhanced by placing the thin metallic film below the array as compared to the SLR mode excited in the array on a glass substrate in the Kretschmann configuration. By taking advantage of interference of the contra-propagating plasmons excited on the metallic film, for certain conditions, which depend on the periodicity of 
the structure, we can excite the Bragg Mode in the array of metallic nanostructures. This mode is similar to the plasmonic modes that can be excited with incidence from the nanostructure side. However, in the Kretschmann configuration the harmonic coupling between the propagating surface plasmon mode of the thin film and the Bragg modes results in a new hybrid mode. This Hybrid Lattice Plasmon (HLP) mode has a strong dispersion, and thus can be tuned effectively over a wide range of excitation wavelengths. The tunability can also be achieved by changing the height of the cylinder (over a range of $1000 \mathrm{~nm}$ in resonance wavelength of the HLP).

Indeed, we have fabricated the nanostructures by electron beam lithography and experimentally confirmed that the HLP mode can be excited using the conventional Kretschmann configuration. The near-field intensity excited in such configurations as well as the mean field intensity distribution was also calculated and proved that excitation of the HLP mode also leads to a significant increase in the mean field intensity around the nanostructures. We can conclude that this type of structure can be used effectively for applications that need such a high confinement of the field intensity, such as SERS and SPR detection.

\section{Methods}

\section{Numerical Simulations.}

For the uniform metallic film we have used the well-known Rouard method, and its generalization to absorbing metallic thin films (see Figure 2) ${ }^{60}$ The refractive indices of gold used for simulations are the values of Johnson and Christy. ${ }^{61}$ For the nanostructures, firstly single cylinders placed at the BK7-Water interface were characterized using the Finite Element Method (FEM) with a perfectly matched layer (PML) surrounding the cylinders. ${ }^{62}$ Then, an array of such cylinders was studied using a numerical model that hybridizes two popular numerical methods: 
FEM, and the periodic Fourier Modal Method (FMM). ${ }^{63}$ This hybrid method gives accurate rigorous results with limited consumption of time and memory. ${ }^{646566}$

The electric and magnetic fields for the structure were calculated for different values of wavelength $(\lambda)$, and different plane incidence wave-vectors using the Hybrid method. $\left(k_{x}=\right.$ $k_{0} n_{b} \sin \theta$, where $k_{0}=2 \pi / \lambda, n_{b}$ the refractive index of the incident medium, and $\theta$ is the incidence angle, as shown in Figure 1). From the calculated fields we derived the value of the absorption of the nanostructures with respect to the incidence EM field.

The idea is to calculate the fields for the periodic array using the FEM method, and then derive the far field effects (reflectivity, transmission, and absorption) using the FMM (see Figure 3).

Finally, a gold film was placed between the cylinder array, and the BK7 layer. Electric field distribution and the consequent far field response were studied using the same hybridized method as mentioned above.

Nanostructure fabrication. BK7 substrates were cleaned in a piranha solution (3:1 ratio of $\mathrm{H}_{2} \mathrm{SO}_{4}(98 \%)$ and $\left.\mathrm{H}_{2} \mathrm{O}_{2}(30 \%)\right)$ for 10 minutes. After a wash step by deionized water, a layer of 3 $\mathrm{nm}$ titanium followed by a layer of $30 \mathrm{~nm}$ gold, were deposited by E-beam evaporation. Then, a layer of around $75 \mathrm{~nm}$ of poly(mehtylmethacrylate) (PMMA) was deposited by spin-coating on glass substrates, and then baked for 15 minutes at $150^{\circ} \mathrm{C}$. Patterns were then obtained by EBL followed by a development step of the exposed regions in a mixture of methylisobutylketone (MIBK), and isopropanol (ISO) (MIBK/ISO ratio: 1:3) for around 90 seconds. After a gold evaporation step, nanocylinders were obtained via a lift-off process carried out by dipping the substrate in acetone for several hours. The obtained structures have a period of around $200 \mathrm{~nm}$, and diameters of around $50 \mathrm{~nm}$ as determined by SEM images (SEMFEG Philips). 
Acknowledgements. The authors acknowledge IDEX Paris Saclay, and ANR P2N (ANR-12NANO-0016) as well as CNANO IDF, Labex NanoSaclay, and LUMAT for partial funding of the project. IOGS/CNRS is also part of the European Network of Excellence in BioPhotonics: Photonics for Life (P4L).

\section{REFERENCES AND NOTES}

1. Barnes, W. L.; Dereux, A.; Ebbesen, T. W., Surface plasmon subwavelength optics. Nature 2003, 424 (6950), 824-830.

2. $\quad$ Schuller, J. A.; Barnard, E. S.; Cai, W. S.; Jun, Y. C.; White, J. S.; Brongersma, M. L., Plasmonics for extreme light concentration and manipulation (vol 9, pg 193, 2010). Nat Mater 2010, 9 (4).

3. Homola, J., Surface plasmon resonance sensors for detection of chemical and biological species. Chem Rev 2008, 108 (2), 462-493.

4. Bardin, F.; Bellemain, A.; Roger, G.; Canva, M., Surface plasmon resonance spectroimaging sensor for biomolecular surface interaction characterization. Biosensors \& Bioelectronics 2009, 24 (7), 2100-2105.

5. Mannelli, I.; Courtois, V.; Lecaruyer, P.; Roger, G.; Millot, M. C.; Goossens, M.; Canva, M., Surface plasmon resonance imaging (SPRI) system and real-time monitoring of DNA biochip for human genetic mutation diagnosis of DNA amplified samples. Sensor Actuat BChem 2006, 119 (2), 583-591.

6. Nakkach, M.; Duval, A.; Ea-Kim, B.; Moreau, J.; Canva, M., Angulo-spectral surface plasmon resonance imaging of nanofabricated grating surfaces. Opt Lett 2010, 35 (13), 22092211.

7. Meyer, S. A.; Auguie, B.; Le Ru, E. C.; Etchegoin, P. G., Combined SPR and SERS Microscopy in the Kretschmann Configuration. J Phys Chem A 2012, 116 (3), 1000-1007.

8. Ropers, C.; Neacsu, C. C.; Elsaesser, T.; Albrecht, M.; Raschke, M. B.; Lienau, C., Grating-coupling of surface plasmons onto metallic tips: A nanoconfined light source. Nano Lett 2007, 7 (9), 2784-2788.

9. $\quad$ Turker, B.; Guner, H.; Ayas, S.; Ekiz, O. O.; Acar, H.; Guler, M. O.; Dana, A., Grating coupler integrated photodiodes for plasmon resonance based sensing. Lab on a Chip 2011, 11 (2), 282-287.

10. Zia, R.; Selker, M. D.; Catrysse, P. B.; Brongersma, M. L., Geometries and materials for subwavelength surface plasmon modes. Journal of the Optical Society of America a-Optics Image Science and Vision 2004, 21 (12), 2442-2446.

11. Myroshnychenko, V.; Rodriguez-Fernandez, J.; Pastoriza-Santos, I.; Funston, A. M.; Novo, C.; Mulvaney, P.; Liz-Marzan, L. M.; de Abajo, F. J. G., Modelling the optical response of gold nanoparticles. Chem Soc Rev 2008, 37 (9), 1792-1805.

12. Jain, P. K.; Eustis, S.; El-Sayed, M. A., Plasmon coupling in nanorod assemblies: Optical absorption, discrete dipole approximation simulation, and exciton-coupling model. J Phys Chem $B$ 2006, 110 (37), 18243-18253. 
13. Cunningham, A.; Muhlig, S.; Rockstuhl, C.; Burgi, T., Coupling of Plasmon Resonances in Tunable Layered Arrays of Gold Nanoparticles. J Phys Chem C 2011, 115 (18), 8955-8960.

14. Wang, Y. Q.; Yan, B.; Chen, L. X., SERS Tags: Novel Optical Nanoprobes for Bioanalysis. Chem Rev 2013, 113 (3), 1391-1428.

15. Ngo, H. T.; Wang, H. N.; Fales, A. M.; Vo-Dinh, T., Label-Free DNA Biosensor Based on SERS Molecular Sentinel on Nanowave Chip. Anal Chem 2013, 85 (13), 6378-6383.

16. Atwater, H. A.; Polman, A., Plasmonics for improved photovoltaic devices. Nat Mater 2010, 9 (3), 205-213.

17. Murphy, C. J.; Gole, A. M.; Stone, J. W.; Sisco, P. N.; Alkilany, A. M.; Goldsmith, E. C.; Baxter, S. C., Gold Nanoparticles in Biology: Beyond Toxicity to Cellular Imaging. Accounts Chem Res 2008, 41 (12), 1721-1730.

18. Mayer, K. M.; Hafner, J. H., Localized Surface Plasmon Resonance Sensors. Chem Rev 2011, 111 (6), 3828-3857.

19. Le, F.; Brandl, D. W.; Urzhumov, Y. A.; Wang, H.; Kundu, J.; Halas, N. J.; Aizpurua, J.; Nordlander, P., Metallic nanoparticle arrays: A common substrate for both surface-enhanced Raman scattering and surface-enhanced infrared absorption. Acs Nano 2008, 2 (4), 707-718.

20. Eustis, S.; El-Sayed, M. A., Why gold nanoparticles are more precious than pretty gold: Noble metal surface plasmon resonance and its enhancement of the radiative and nonradiative properties of nanocrystals of different shapes. Chem Soc Rev 2006, 35 (3), 209-217.

21. Juan, M. L.; Righini, M.; Quidant, R., Plasmon nano-optical tweezers. Nat Photonics 2011, 5 (6), 349-356.

22. Wang, X. L.; Gogol, P.; Cambril, E.; Palpant, B., Near- and Far-Field Effects on the Plasmon Coupling in Gold Nanoparticle Arrays. J Phys Chem C 2012, 116 (46), 24741-24747.

23. Koenderink, A. F., Plasmon Nanoparticle Array Waveguides for Single Photon and Single Plasmon Sources. Nano Lett 2009, 9 (12), 4228-4233.

24. Li, J. T.; Cushing, S. K.; Zheng, P.; Meng, F. K.; Chu, D.; Wu, N. Q., Plasmon-induced photonic and energy-transfer enhancement of solar water splitting by a hematite nanorod array. Nat Commun 2013, 4.

25. Khoury, C. G.; Norton, S. J.; Vo-Dinh, T., Plasmonics of 3-D Nanoshell Dimers Using Multipole Expansion and Finite Element Method. Acs Nano 2009, 3 (9), 2776-2788.

26. Live, L. S.; Dhawan, A.; Gibson, K. F.; Poirier-Richard, H. P.; Graham, D.; Canva, M.; Vo-Dinh, T.; Masson, J. F., Angle-dependent resonance of localized and propagating surface plasmons in microhole arrays for enhanced biosensing. Analytical and Bioanalytical Chemistry 2012, 404 (10), 2859-2868.

27. Yu, Q. M.; Guan, P.; Qin, D.; Golden, G.; Wallace, P. M., Inverted size-dependence of surface-enhanced Raman scattering on gold nanohole and nanodisk arrays. Nano Lett 2008, 8 (7), 1923-1928.

28. Zhou, F.; Liu, Y.; Cai, W. P., Huge local electric field enhancement in hybrid plasmonic arrays. Opt Lett 2014, 39 (5), 1302-1305.

29. Lodewijks, K.; Ryken, J.; Van Roy, W.; Borghs, G.; Lagae, L.; Van Dorpe, P., Tuning the Fano Resonance Between Localized and Propagating Surface Plasmon Resonances for Refractive Index Sensing Applications. Plasmonics 2013, 8 (3), 1379-1385.

30. Zou, S. L.; Schatz, G. C., Silver nanoparticle array structures that produce giant enhancements in electromagnetic fields. Chem Phys Lett 2005, 403 (1-3), 62-67.

31. Auguie, B.; Barnes, W. L., Diffractive coupling in gold nanoparticle arrays and the effect of disorder. Opt Lett 2009, 34 (4), 401-403. 
32. Väkeväinen, A. I.; Moerland, R. J.; Rekola, H. T.; Eskelinen, A. P.; Martikainen, J. P.; Kim, D. H.; Törmä, P., Plasmonic Surface Lattice Resonances at the Strong Coupling Regime. Nano Lett 2013.

33. Lovera, A.; Gallinet, B.; Nordlander, P.; Martin, O. J. F., Mechanisms of Fano Resonances in Coupled Plasmonic Systems. Acs Nano 2013, 7 (5), 4527-4536.

34. Gallinet, B.; Martin, O. J. F., Refractive Index Sensing with Subradiant Modes: A Framework To Reduce Losses in Plasmonic Nanostructures. Acs Nano 2013, 7 (8), 6978-6987.

35. Aubry, A.; Lei, D. Y.; Maier, S. A.; Pendry, J. B., Plasmonic Hybridization between Nanowires and a Metallic Surface: A Transformation Optics Approach. Acs Nano 2011, 5 (4), 3293-3308.

36. Papanikolaou, N., Optical properties of metallic nanoparticle arrays on a thin metallic film. Physical Review B 2007, 75 (23).

37. Lassiter, J. B.; McGuire, F.; Mock, J. J.; Ciraci, C.; Hill, R. T.; Wiley, B. J.; Chilkoti, A.; Smith, D. R., Plasmonic Waveguide Modes of Film-Coupled Metallic Nanocubes. Nano Lett 2013, 13 (12), 5866-5872.

38. Zuloaga, J.; Prodan, E.; Nordlander, P., Quantum Description of the Plasmon Resonances of a Nanoparticle Dimer. Nano Lett 2009, 9 (2), 887-891.

39. Davis, T. J.; Gomez, D. E.; Vernon, K. C., Simple Model for the Hybridization of Surface Plasmon Resonances in Metallic Nanoparticles. Nano Lett 2010, 10 (7), 2618-2625.

40. Forestiere, C.; Dal Negro, L.; Miano, G., Theory of coupled plasmon modes and Fanolike resonances in subwavelength metal structures. Physical Review B 2013, 88 (15).

41. Miroshnichenko, A. E.; Flach, S.; Kivshar, Y. S., Fano resonances in nanoscale structures. Rev Mod Phys 2010, 82 (3), 2257-2298.

42. Luk'yanchuk, B.; Zheludev, N. I.; Maier, S. A.; Halas, N. J.; Nordlander, P.; Giessen, H.; Chong, C. T., The Fano resonance in plasmonic nanostructures and metamaterials. Nat Mater 2010, 9 (9), 707-715.

43. Francescato, Y.; Giannini, V.; Maier, S. A., Plasmonic Systems Unveiled by Fano Resonances. Acs Nano 2012, 6 (2), 1830-1838.

44. Gantzounis, G.; Stefanou, N.; Papanikolaou, N., Optical properties of periodic structures of metallic nanodisks. Physical Review B 2008, 77 (3).

45. Butt, H.; Dai, Q.; Rajesekharan, R.; Wilkinson, T. D.; Amaratunga, G. A. J., Plasmonic Band Gaps and Waveguide Effects in Carbon Nanotube Arrays Based Metamaterials. Acs Nano 2011, 5 (11), 9138-9143.

46. Raether, H., Surface-Plasmons on Smooth and Rough Surfaces and on Gratings. Springer Tr Mod Phys 1988, 111, 1-133.

47. Li, Y.; Zhao, K.; Sobhani, H.; Bao, K.; Nordlander, P., Geometric Dependence of the Line Width of Localized Surface Plasmon Resonances. J Phys Chem Lett 2013, 4 (8), 13521357.

48. Ni, W. H.; Ambjornsson, T.; Apell, S. P.; Chen, H. J.; Wang, J. F., Observing PlasmonicMolecular Resonance Coupling on Single Gold Nanorods. Nano Lett 2010, 10 (1), 77-84.

49. Carron, K. T.; Fluhr, W.; Meier, M.; Wokaun, A.; Lehmann, H. W., Resonances of TwoDimensional Particle Gratings in Surface-Enhanced Raman-Scattering. J Opt Soc Am B 1986, 3 (3), 430-440.

50. Rodriguez, S. R. K.; Abass, A.; Maes, B.; Janssen, O. T. A.; Vecchi, G.; Rivas, J. G., Coupling Bright and Dark Plasmonic Lattice Resonances. Phys Rev X 2011, 1 (2). 
51. Kravets, V. G.; Schedin, F.; Grigorenko, A. N., Extremely narrow plasmon resonances based on diffraction coupling of localized plasmons in arrays of metallic nanoparticles. Phys Rev Lett 2008, 101 (8).

52. Barnes, W. L.; Preist, T. W.; Kitson, S. C.; Sambles, J. R., Physical origin of photonic energy gaps in the propagation of surface plasmons on gratings. Physical Review B 1996, 54 (9), 6227-6244.

53. Novotny, L., Strong coupling, energy splitting, and level crossings: A classical perspective. Am J Phys 2010, 78 (11), 1199-1202.

54. Kelf, T. A.; Sugawara, Y.; Baumberg, J. J.; Abdelsalam, M.; Bartlett, P. N., Plasmonic band gaps and trapped plasmons on nanostructured metal surfaces. Phys Rev Lett 2005, 95 (11).

55. Okamoto, T.; Simonen, J.; Kawata, S., Plasmonic band gaps of structured metallic thin films evaluated for a surface plasmon laser using the coupled-wave approach. Physical Review $B$ 2008, 77 (11).

56. Chamtouri, M.; Sarkar, M.; Moreau, J.; Besbes, M.; Ghalila, H.; Canva, M., Field enhancement and target localization impact on the biosensitivity of nanostructured plasmonic sensors. J. Opt. Soc. Am. B 2014, 31 (5), 1223-1231.

57. Moskovits, M., Surface-enhanced Raman spectroscopy: a brief perspective. Top Appl Phys 2006, 103, 1-17.

58. Liu, Y.; Xu, S. P.; Tang, B.; Wang, Y.; Zhou, J.; Zheng, X. L.; Zhao, B.; Xu, W. Q., Note: Simultaneous measurement of surface plasmon resonance and surface-enhanced Raman scattering. Rev Sci Instrum 2010, 81 (3).

59. Sereda, A.; Moreau, J.; Canva, M.; Maillart, E., High performance multi-spectral interrogation for surface plasmon resonance imaging sensors. Biosensors and Bioelectronics 2014, 54 (0), 175-180.

60. Lecaruyer, P.; Maillart, E.; Canva, M.; Rolland, J., Generalization of the Rouard method to an absorbing thin-film stack and application to surface plasmon resonance. Applied Optics 2006, 45 (33), 8419-8423.

61. Johnson, P. B.; Christy, R. W., Optical Constants of the Noble Metals. Physical Review B 1972, 6 (12), 4370-4379.

62. Dossou, K.; Byrne, M. A.; Botten, L. C., Finite element computation of grating scattering matrices and application to photonic crystal band calculations. Journal of Computational Physics 2006, 219 (1), 120-143.

63. Moharam, M. G.; Grann, E. B.; Pommet, D. A.; Gaylord, T. K., Formulation for Stable and Efficient Implementation of the Rigorous Coupled-Wave Analysis of Binary Gratings. Journal of the Optical Society of America a-Optics Image Science and Vision 1995, 12 (5), 10681076.

64. Besbes, M.; Hugonin, J. P.; Lalanne, P.; van Haver, S.; Janssen, O. T. A.; Nugrowati, A. M.; Xu, M.; Pereira, S. F.; Urbach, H. P.; van de Nes, A. S.; Bienstman, P.; Granet, G.; Moreau, A.; Helfert, S.; Sukharev, M.; Seideman, T.; Baida, F. I.; Guizal, B.; Van Labeke, D., Numerical analysis of a slit-groove diffraction problem. Journal of the European Optical Society-Rapid Publications 2007, 2.

65. Hugonin, J. P.; Besbes, M.; Lalanne, P., Hybridization of electromagnetic numerical methods through the G-matrix algorithm. Opt Lett 2008, 33 (14), 1590-1592.

66. Sarkar, M.; Chamtouri, M.; Moreau, J.; Besbes, M.; Canva, M., Introducing 2D confined propagating plasmons for surface plasmon resonance sensing using arrays of metallic ribbons. Sensor Actuat B-Chem 2014, 191, 115-121. 
